\title{
Robespierre dans les publications françaises et anglophones depuis l'an 2000
}

Robespierre in French and English publications since the year 2000

Marc Belissa et Julien Louvrier

\section{(2) OpenEdition \\ 1 Journals}

Édition électronique

URL : https://journals.openedition.org/ahrf/12687

DOI : $10.4000 / a h r f .12687$

ISSN : 1952-403X

Éditeur :

Armand Colin, Société des études robespierristes

Édition imprimée

Date de publication : 1 mars 2013

Pagination : 73-93

ISBN : 978-2-200-92824-7

ISSN : 0003-4436

Référence électronique

Marc Belissa et Julien Louvrier, «Robespierre dans les publications françaises et anglophones depuis l'an $2000 »$ ", Annales historiques de la Révolution française [En ligne], 371 | janvier-mars 2013, mis en ligne le 01 mars 2016, consulté le 01 juillet 2021. URL : http://journals.openedition.org/ahrf/12687 DOl : https://doi.org/10.4000/ahrf.12687 


\title{
ROBESPIERRE DANS LES PUBLICATIONS FRANCAISES ET ANGLOPHONES DEPUIS L'AN 2000
}

Marc BELISSA et Julien LOUVRIER

\begin{abstract}
En ce qui concerne Robespierre, le « moment » du Bicentenaire s'étend bien au-delà de 1989. II se termine en réalité en 1997 ou 1998 quand paraissent les derniers colloques conçus dans les années qui ont suivi les commémorations. Cet article s'intéresse aux publications sur Robespierre dans la période qui suit le « moment " du Bicentenaire jusqu'à 2012. Elle commence grosso modo en l'an 2000 et s'achève (provisoirement) avec les suites de l'affaire de l'achat par l'État des manuscrits de Robespierre et de Lebas chez Sotheby's. Cette courte période est-elle marquée par un « retour » à Robespierre ? Quelles sont les grandes caractéristiques des publications francophones et anglophones, universitaires et académiques sur Maximilien Robespierre?
\end{abstract}

Mots-clés : Robespierre, historiographie.

Figure à la fois largement occultée dans les célébrations commémoratives, et pourtant toujours présente en creux dans les discours officiels, Robespierre a été le grand absent du Bicentenaire de la Révolution française. Les deux ouvrages de référence qui se sont intéressés au Bicentenaire comme objet d'histoire ${ }^{1}$ ont, chacun avec leur propre démarche, analysé les enjeux de cette absence/présence virtuelle selon les acteurs des commémorations. Volontairement ignoré dans les célébrations officielles parce qu'il était censé représenter la face sanglante de la Révolution dont François

(1) Steven KAPLAN, Adieu 89, Paris, Fayard, 1993 ; Patrick GARCIA, Le Bicentenaire de la Révolution française : pratiques sociales d'une commémoration, Paris, CNRS, 2000. 
Mitterrand ne voulait à aucun prix qu'elle soit associée aux festivités de la commémoration, Robespierre a également été escamoté (à quelques exceptions individuelles près) par la gauche socialiste. Dans leur volonté de diaboliser et de flétrir l'ensemble de la Révolution française comme un processus inutile, destructeur et anti-français, l'extrême-droite, les « anti-89 » et la fraction « vendéenne » des anti-révolutionnaires n'ont, quant à eux, pas particulièrement porté leur effort polémique sur la figure de Robespierre, préférant insister sur la Terreur comme phénomène globalisant (censé être présent dès 1789). La droite parlementaire ne s'est pas plus concentrée sur Maximilien, occupée qu'elle était à dénoncer la commémoration comme une manœuvre mitterrandienne et à dépolitiser les fêtes prévues dans le cadre de la ville de Paris, alors dirigée par Jacques Chirac. L'école de François Furet et ses prolongements à l'EHESS et dans les médias n'ont accordé qu'un intérêt tout à fait secondaire à la figure de Robespierre, considéré finalement plus comme une incarnation du discours jacobin que comme un acteur doté d'une pensée propre.

Finalement, seuls les historiens communistes comme Claude Mazauric et Michel Vovelle, des associations locales comme les Amis de Robespierre pour le Bicentenaire de la Révolution ou nationales comme la Société des études robespierristes, mais aussi des chercheurs comme Françoise Brunel, Florence Gauthier, Jacques Guilhaumou ${ }^{2}$, ou le philosophe Georges Labica ${ }^{3}$ pour n'en citer que quelques-uns, se sont emparés dans les commémorations ou dans leurs travaux - avec des approches historiographiques diverses - de la figure de Robespierre dans une volonté de réhabilitation de la part radicale et sociale de la Révolution française. Défendre la mémoire de Robespierre et/ou étudier son action dans une démarche contextuelle contre les falsifications était une manière d'affirmation des potentialités contemporaines de l'idée même de Révolution et des droits de l'homme et du citoyen.

Absent des commémorations, Robespierre se rattrape en quelque sorte dans la «queue du Bicentenaire » pour paraphraser l'expression thermidorienne. Ce sont essentiellement dans les colloques conçus dans la période du Bicentenaire, mais qui, pour des raisons diverses, ne se sont tenus qu'au début des années 1990 (et qui pour certains n'ont été publiés qu'en 1997) que la figure de Robespierre a suscité une série de contributions

(2) Françoise BrunEl, Thermidor, Bruxelles, Complexe, 1989 ; Jacques GuILHAUMOU, La langue politique de la Révolution française, Paris, Klincksieck, 1989 ; Florence GAUTHIER, Triomphe et mort du droit naturel en Révolution, Paris, PUF, 1992.

(3) Georges LABICA, Robespierre, une politique de la philosophie, Paris, PUF, 1990. 
historiographiques universitaires. Ainsi, les colloques d'Arras, de Naples, d'Amsterdam et de Winchester ont cherché à combler le vide béant créé par l'occultation de Robespierre dans les commémorations ${ }^{4}$. En simplifiant quelque peu, les deux colloques d'Arras et de Naples se situaient plutôt dans le cadre de l'école dite « jacobine » ou « jauressienne », celui d'Amsterdam (à un degré moindre) et surtout celui de Winchester plutôt dans celui de l'école d'inspiration furétienne.

Le «moment » du Bicentenaire s'étend donc, en ce qui concerne Robespierre, bien au-delà de 1989, il se termine en réalité en 1997 ou 1998. Cet article s'intéresse à la production dans la période qui suit le "moment » du Bicentenaire jusqu'à 2012. Elle commence grosso modo en l'an 2000 et s'achève (provisoirement ?) avec les suites de l'affaire de l'achat par l'État des manuscrits de Robespierre et de Lebas chez Sotheby's qui a suscité un débat public intéressant dont la contribution de Serge Aberdam, Jean-Paul Rothiot et Cyril Triolaire rend compte dans ce même numéro de la revue. Cette courte période est-elle marquée par un « retour » à Robespierre ? Le contexte intellectuel peut sembler l'indiquer. La pièce Notre Terreur donnée au Théâtre de la Colline en 2009, l'initiative du groupe du Front de Gauche au conseil municipal de Paris demandant une rue Robespierre dans la capitale, les apostrophes de Laurence Parisot et du philosophe médiatique Michel Onfray contre les références à l'Incorruptible dans le discours du candidat à la présidentielle Jean-Luc Mélenchon, une émission intitulée Robespierre, bourreau de la Vendée ? sur les antennes de la télévision publique : autant d'éléments qui montrent que la figure de Maximilien fait toujours clivage, au moins dans le paysage politique français. Qu'en est-il du côté des publications ?

Nous avons choisi de limiter notre corpus aux travaux qui traitent explicitement de Robespierre, excluant les histoires générales de la Révolution dans lesquelles il n'apparaît que comme un acteur parmi d'autres (avec une place souvent assez réduite d'ailleurs). Ce corpus pour lequel nous nous sommes limités aux textes en français et en anglais (mais une recherche rapide a montré qu'il y en a très peu dans d'autres langues) et dont nous avons exclu les rééditions d'ouvrages antérieurs et les thèses non-publiées - se compose d'un peu moins d'une trentaine

(4) Jean-Pierre JESSENE et al. Robespierre de la Nation artésienne à la République et aux Nations, Villeneuve d'Ascq, 1993 ; Jean EHRARD (dir.) Images de Robespierre, Napoli, Vivarium, 1996 ; Annie JOURDAN (dir.), Robespierre : figure-réputation, Amsterdam, Rodopi, 1996 ; Colin HAYDOn, William DoYle (dir.), Robespierre, Cambridge, Cambridge University Press, 1999. 
d'articles, d'ouvrages ou de chapitres d'ouvrages. Nous avons clos cette bibliographie avec l'ouvrage de Cécile Obligi paru en $2012^{5}$.

On peut diviser cette production en deux grandes catégories très subjectives. Tout d'abord, les ouvrages d'écrivains plus ou moins spécialisés dans le domaine historique, mais qui se situent, pour des raisons diverses, en dehors de l'histoire universitaire proprement dite. Il s'agit le plus souvent de livres qui présentent une vision négative de l'homme Robespierre et de son action - et presque toujours marquée par les présupposés de la pensée contre-révolutionnaire modernisée sous la forme de «l'anti-totalitarisme $»^{6}$. Une deuxième catégorie regroupe les travaux authentiquement universitaires dans différents domaines (histoire, sciences politiques, psychanalyse), nous y avons adjoint les anthologies de textes de Robespierre édités et présentés par des universitaires ${ }^{7}$.

Ces textes sont évidemment marqués par des approches différentes en fonction des objectifs de leurs auteurs, mais aussi en raison des conditions

(5) Nous n'avons pas pu prendre en compte ici l'ouvrage collectif réalisé sous l'égide de la SER parue en octobre 2012 sous le titre Robespierre, portraits croisés, Paris, A. Colin, 2012.

(6) Jean ARTARIT, Robespierre ou L'impossible filiation, Paris, la Table ronde, 2003 ; Laurent DINGLI, Robespierre, Paris, Flammarion, 2004, Jean-François FAYARD, Les 100 jours de Robespierre, Paris, Grancher, 2005, Joël SCHMIDT, Robespierre, Paris, Gallimard, 2011. On peut y ajouter le numéro 777 de la revue Historia publié en septembre 2011 dont le titre indique assez la ligne éditoriale :

«Robespierre : un psychopathe légaliste » (bien que certains articles de ce numéro contredisent totalement cette formule). Précisons que Jean-François Fayard et Laurent Dingli sont bien docteurs en histoire mais n'enseignent pas dans les universités.

(7) Biographies; Ruth SCURR, Fatal purity : Robespierre and the French Revolution, London, Chatto \& Windus, 2006; Peter MCPHEE, Robespierre. A Revolutionary Life, Yale University Press, 2012; Cécile OBLIGI, Robespierre. La probité révoltante, Paris, Belin, 2012.

Éditions de textes : Pour le bonheur et pour la liberté : discours, choix et présentation par Yannick Bosc, Florence GAUTHIER et Sophie WAHNICH, Paris, Éd. la Fabrique, 2000, Marie-Laure LEGAY, Robespierre et le pouvoir provincial : dénonciation \& émancipation politique. A la nation artésienne : sur la nécessité de réformer les Etats d'Artois, édition critique, Arras, 2001 ; Discours sur la religion, la république, l'esclavage, La Tour d'Aigues, Éd. de l'Aube, 2006, Euvres de Maximilien Robespierre. Tome XI, Compléments (1784-1794), éd. présentée et annotée par Florence GAUTHIER, Paris, Société des études robespierristes, 2007 ; Robespierre, entre vertu et terreur, introduction et présentation de Slavoj ŽIŽEK, Paris, Stock, 2008.

Articles de revues ou chapitres d'ouvrages universitaires :

Jean-Daniel PIQUET, « Robespierre et la liberté des Noirs en l'an II d'après les archives des comités et les papiers de la commission Courtois », AHRF, n 323, 2001, p. 66-91 ; Jean-François JACOUTY, « Robespierre selon Louis Blanc », AHRF, n 331, 2003, p. 105-127 ; Antoine de BAECQUE, Les duels politiques : de Danton-Robespierre à Royal-Sarkozy, Paris, Hachette, 2007 ; Maxime Rosso, « Les réminiscences spartiates dans les discours et la politique de Robespierre de 1789 à thermidor », AHRF n³49, 2007, p. 51-78; Jacques GuILHAUMOU, « Robespierre et la formation de l'esprit politique au cours des années 1780. Pour une ontologie historique du discours robespierriste », Mots, les langages du politique, $\mathrm{n}^{\circ}$ 89, 2009 ; Alyssa SEPINWALL, « Robespierre, Old Regime Feminist ? Gender, the Late Eighteenth Century, and the French Revolution Revisited », The Journal of Modern History, 82, 1, mars 2010, p. 1-29; David ANDRESS; « Living the Revolutionary Melodrama: Robespierre's Sensibility and the Construction of Political Commitment in the French Revolution », Representations, Berkeley, Spring 2011, p. 103-128. 
éditoriales de leur production, néanmoins, nous avons pu déceler des points communs dans les ouvrages de la première catégorie. Premièrement, ces publications partent du postulat qu'il n'y a rien de nouveau à apporter à la connaissance érudite de la vie et de l'action de Robespierre, mais qu'il s'agit d'apporter une interprétation de la figure de Robespierre en tant qu'incarnation de la Révolution. Ces travaux utilisent largement des sources thermidoriennes et post-révolutionnaires sans prendre en compte les effets de construction de la légende noire, de la légende « dorée » des robespierristes de la génération romantique, et surtout sans effectuer le travail minutieux de décryptage du cheminement des lieux communs et de la sédimentation des interprétations depuis deux siècles. Pour ne prendre qu'un exemple, nombreux sont les auteurs qui se contentent de répéter les anecdotes sur Robespierre présentes chez l'abbé Proyart ou Charlotte Robespierre sans les analyser dans le contexte de leur production. Or ces anecdotes ne peuvent être sérieusement utilisées en faisant l'économie d'une réflexion méthodologique sur les objectifs de leur construction. Par ailleurs, en interprétant Robespierre, ces publications tendent à le décontextualiser. Enfin, une des constantes des travaux sur Robespierre publiés depuis une quinzaine d'années est le retour d'une vision psychologique, voire pathologique, du phénomène révolutionnaire qui se réduit à une analyse de l'action de Robespierre par des interprétations qui relèvent de la caractérologie du XIX ${ }^{\mathrm{e}}$ siècle, relookées par une certaine forme de discours psychanalytique.

C'est, dans un premier temps, autour de ces quelques thèmes que nous souhaitons analyser ici cette production, avant de nous consacrer aux apports des travaux historiographiques universitaires proprement dits.

\section{Robespierre dans les ouvrages non-universitaires}

Ce qui caractérise la plupart des ouvrages « grand public » consacrés à Robespierre dans la période considérée est qu'ils sont le fait de nonspécialistes (Dingli, Schmidt) ou de non-historiens (Artarit). Nous avons à faire à des auteurs qui s'affranchissent volontiers des méthodes de lecture de sources et des problématiques actuelles présentes dans le champ académique. Recourant fréquemment à la téléologie, donnant volontiers dans l'illusion biographique, utilisant abondamment les anecdotes les plus controuvées, mais aussi les Euvres de Robespierre comme un corpus décontextualisé, ils n'hésitent pas à spéculer, à broder, pour asseoir leurs Robespierre. L'historien moderniste est souvent effaré par des erreurs factuelles et des interprétations qui montrent assez qu'une partie de 
ces auteurs n'a qu'une connaissance superficielle de la période dont ils parlent. Inutile de dresser ici un catalogue, mais la palme en ce domaine revient peut-être à la biographie de Joël Schmidt remplie d'erreurs et de confusions inquiétantes ${ }^{8}$. Même quand l'érudition semble présente via une copieuse bibliographie et des notes de bas de page, comme chez Dingli, il s'agit plus d'une érudition-alibi que d'un véritable travail sur les sources ${ }^{9}$. Il est vrai qu'Artarit, Dingli ou Schmidt vouent un solide mépris aux historiens de l'école dite classique ou « jacobine » de la Révolution (histoire « cadenassée », « fossilisée » chez Artarit ${ }^{10}$, « formules doctes et froides dispensée sur des bancs d'école » chez Dingli) et ne semblent jurer que par les travaux de François Furet, même si celui-ci aurait été sans doute fort étonné de ce qu'on lui fait dire, s'il était encore en vie. Rappelons que, malgré bien des contradictions, Furet a toujours refusé de voir dans Robespierre le père des totalitarismes du $\mathrm{XX}^{\mathrm{e}}$ siècle et n'a jamais accepté les comparaisons entre Robespierre et Staline (et encore moins avec Hitler). Pour Furet, Robespierre « incarne » la Terreur, il ne l'invente pas, ni ne la dirige $^{11}$. La référence à Furet dans les travaux anti-robespierristes récents est donc tout sauf précise...

Nous reviendrons plus loin sur le thème du " pathologique », dominant dans les ouvrages d'Artarit, Dingli ou Schmidt, mais ces biographies ont en commun un biais interprétatif qui n'a rien de nouveau et qui se contente de reprendre les lieux communs de la littérature contrerévolutionnaire depuis deux siècles : Robespierre est un personnage inquiétant ou du moins peu équilibré, un raté aigri de la société d'Ancien Régime, un homme plutôt médiocre, mais suffisamment tenace, rigide et rancunier pour suivre la vague révolutionnaire et l'utiliser à son profit pour éliminer ses adversaires politiques et parvenir au « pouvoir suprême » (?), un utopiste prêt à tout pour réaliser un idéal de vertu par l'élimination physique de tous ceux qui s'y opposent... Il est surtout l'homme de la « Terreur », présentée sous la forme simpliste d'une violence d'État et de masse.

Ces ouvrages partagent également l'idée qu'il n'y a rien de neuf à apprendre sur Robespierre. Sa vie, et sa pensée politique, lorsqu'on suppose qu'il en a une, ont déjà été examinées sous tous les angles

(8) La Guerre de Sept Ans met aux prises la France contre une coalition austro-prussienne, Robespierre ne connaît pas le droit français, car on ne lui a enseigné que le droit romain, les Oratoriens enseignent Rousseau et Mably dans leurs collèges, etc.

(9) Voir Claude MAZAURIC, « Robespierre ou l'impossible filiation », AHRF n³39, janviermars 2005, p. 155-159.

(10) Jean ARTARIT, op. cit., p. IX.

(11) François FURET, Penser la Révolution française, Paris, Folio histoire, 1978, p. 94-102. 
possibles (c'est un « disciple de Rousseau »). La tâche du biographe donc réside essentiellement dans le dosage, c'est-à-dire l'importance qu'il décide d'accorder à tel ou tel facteur ou moment dans la vie de Robespierre. Domine alors une «méthode » à la fois régressive et téléologique : Robespierre est condamné d'avance au regard du résultat connu de la « Terreur ». Chaque événement de son existence, chacune de ses prises de position est analysée non comme une adaptation à une situation ou à un problème politique ou social donnés, mais en fonction d'une vision d'ensemble aboutissant à la « Terreur», au «pouvoir » et à la mort. Inutile de préciser qu'une telle approche est rigoureusement opposée à toute démarche épistémologique qui implique à la fois une réflexion sur la biographie et surtout sur le statut des sources. Les ragots les plus éculés de la légende noire thermidorienne et postérieure sont utilisés, certes, avec parfois quelques précautions oratoires, mais sans aucune analyse. Ces ouvrages effectuent tout de même un tri parmi les éléments de la légende. Les outrances les plus voyantes sont éliminées (la tannerie de peau humaine pour fournir des souliers aux sans-culottes de l'abbé Proyart par exemple), mais combien d'autres sont présentées comme des faits certains ou possibles (le «témoignage » de Villiers, le prétendu secrétaire de Robespierre en 1790 par exemple)... Ce tri résulte du fait que ce n'est pas tant la figure du «monstre sanguinaire » thermidorien qu'il s'agit de mettre en valeur que celle de l'idéologue froid, utopiste et mortifère. C'est à partir de ce présupposé que l'on admet ou non la « validité » de tel ou tel témoignage.

Ces approches purement idéologiques aboutissent paradoxalement à une dépolitisation des enjeux et à une décontextualisation des étapes de la vie de Robespierre, avec ses errements, ses revirements, ses surprises, ses circonstances, la reconfiguration permanente de l'horizon des possibles dans la dynamique révolutionnaire, etc., au profit d'un discours figé qui n'évolue que dans une logique d'ambition et de pouvoir. Ces travaux s'apparentent donc davantage à un discours idéologique désincarné (sur Robespierre, sur la Révolution en général, sur le changement social, sur le stalinisme, le communisme parfois) qu'à une histoire de la Révolution. Ils ne font pas l'histoire de Robespierre, ils élaborent un discours idéologique à partir de la « biographie » de Robespierre.

Entendons-nous bien, le fait que des non-spécialistes écrivent sur la Révolution et Robespierre n'est évidemment pas un problème en soi. La Révolution est un sujet de recherche, mais aussi un objet civique qu'il n'est pas question de "réserver» au monopole universitaire historique. Qu'un psychiatre, un romancier ou tout citoyen écrivent ce qui leur plaît sur Robespierre ne nous choque en rien, mais ils doivent le faire avec 
les méthodes historiques s'ils veulent être lus en historiens et non en pamphlétaires ou en essayistes. Or ces ouvrages tournent le dos à toute réflexion méthodologique et historiographique. D'où notre distinction stricte entre les travaux des historiens universitaires, qui se soumettent à la critique de leurs pairs et aux règles de la profession, et les ouvrages qui se situent en dehors du champ académique et qui échappent par définition au débat critique et à la confrontation avec les spécialistes.

\section{Le retour du pathologique : Robespierre psychopathe}

Les interprétations qui font de Robespierre un malade mental et qui expliquent son parcours politique par des considérations psychologiques ou pathologiques n'ont rien de nouveau. Elles ont fait un retour spectaculaire dans le moment du Bicentenaire après une éclipse - relative - entre la fin des années 1930 et les années 1970. Mais il semble qu'à la suite des diatribes anti-robespierristes du Bicentenaire, elles se maintiennent aujourd'hui, surtout, bien entendu, dans les publications ouvertement antirévolutionnaires, mais aussi, parfois à un degré moindre, dans nombre d'ouvrages de vulgarisation.

On retrouve ainsi abondamment le thème de la personnalité pathologique et asexuelle de Robespierre dans les ouvrages récents d'Eli Sagan, de Jean Artarit, de Laurent Dingli ou de Joël Schmidt, thèmes souvent associés à un « anti-totalitarisme » simpliste qui fait des grands dictateurs du $\mathrm{XX}^{\mathrm{e}}$ siècle des malades mentaux. Ces ouvrages ont en commun une vision de Robespierre comme un fanatique paranoïaque porté au pouvoir par l'événement révolutionnaire, et maître d'œuvre d'une politique totalitaire, de terreur et de mort au nom d'idéaux absurdes et chimériques.

Il est inutile de multiplier à l'excès les citations de ces ouvrages qui disent souvent la même chose, quelques exemples suffiront...

En 2011, le psychanalyste et sociologue Eli Sagan consacre un chapitre de son Citizens and Cannibals à Robespierre ${ }^{12}$. Ce chapitre intitulé sobrement « Robespierre, virtuous, paranoid, narcissist, dictator, genius of moral critique, terrorist $»-$ explique que l'aspiration à la vertu chez Robespierre est un comportement fondamentalement pathologique et narcissique qui « à un stade complexe » assume une dimension paranoïaque. Robespierre souffre donc de deux perversions premières : «the narcissism

(12) Eli SAGAN, Citizens and Cannibals, The French Revolution, The Struggle for Modernity and The Origins of Ideological Terror, Lanham, Rowan and Littlefield, 2001. 
of virtue and the paranoid grandiosity of moral critique $»^{13}$. De là découlent une « psyché clivée » et une obsession du sacrifice qui explique sa défaite. Celle-ci n'est donc pas due à ses adversaires ou à ses contradictions politiques, mais a été provoquée par sa propre psychopathologie ${ }^{14}$.

Simple mise en bouche... car avec Jean Artarit, le psychanalyste et historien autoproclamé des guerres de Vendée, on passe à un stade supérieur dans l'analyse psychopathologique de Robespierre. Dans un ouvrage de plus de 500 pages (publié en 2003 et réédité par le CNRS), Artarit entend suivre la trajectoire de Robespierre « dans une démarche historique classique mais avec le regard du psychiatre et psychanalyste ${ }^{15}$. On cherchera vainement une critique historienne des sources thermidoriennes et postérieures de la légende noire, puisque les témoignages des adversaires de Robespierre ne sont pas des «injures » sous forme d'accusation de folie, «mais bien la plupart du temps [des] observations souvent fines, sur la psychologie inquiétante de l'homme et son évidente dimension pathologique $»^{16}$. À ce niveau «d'évidence », la critique historique est bien obligée de se tenir coite... L'ensemble de l'ouvrage n'est qu' une longue suite d'interprétations psychanalytiques des thèmes de la légende noire, sans autre recul critique qu'une série d'atténuations des topoi empruntés à l'abbé Proyart, Montjoie, et autres écrivains contre-révolutionnaires, assaisonnés de pseudo-emprunts à François Furet : Proyart est «plus crédible qu'on a bien voulu l'accepter ${ }^{17}$, Montjoie aussi, mais quand même..., etc.

À la base de cette interprétation, l'idée de « l'impossible filiation ». Le père de Robespierre ayant abandonné ses enfants, il en découle une série de dysfonctionnements mentaux qui aboutissent à une personnalité « évidemment » pathologique, expliquant dans un bel exemple de téléologie l'action de Robespierre dans la Révolution. Jean Artarit décèle chez Maximilien «l'existence d'un état passionnel de persécution, associé à des idées de toute-puissance qui a rapidement fait l'unanimité chez les observateurs ». Cette « autodestruction narcissique, projetée dans le politique, est, à l'évidence [sic] » à l'origine de la Terreur.

«Tout le ressort» de la vie de Maximilien est l'absence de son père $^{18}$. La tristesse du jeune Robespierre évoquée dans les mémoires de Charlotte

(13) Ibid, p. 485.

(14) Ibid., p. 491.

(15) Jean ARTARIT, op. cit., p. IX.

(16) Ibid, p. X.

(17) Ibid., p. 43.

(18) Ibid., p. 18. 
« n'est ni plus ni moins qu'une fuite quasi-permanente dans une rêverie de compensation, sorte d'hallucination du désir, où il n'est pas difficile de deviner (sic) que l'enfant s'imaginait aux côtés de sa mère vivante, et où il se campait aussi dans le rôle d'un personnage tout-puissant et sans faille, annulant ou au contraire avalisant la faiblesse et la trahison de son père ${ }^{19}{ }$.

L'anecdote cent fois répétée des pigeons du petit Maximilien devient également un grave symptôme, car l'amour des oiseaux est lié à la capacité d'avoir des activités sexuées ${ }^{20}$. Ce qui fascine en fait Robespierre, c'est la vie en cage et la capacité de faire du mal aux oiseaux : «il n'est pas absolument [sic] certain que le petit Maximilien ait exercé des violences sur les oiseaux, mais il ne fut certainement [re-sic] pas l'innocent que décrit sa sœur ».

Arrivé à ce point de « l'analyse », l'oiseau-pénis pose problème. Pour lutter contre le « désespoir engendré par cette impasse dans sa maturation psychologique », «on » conçoit que l'enfant se soit révolté et ait pu retourner ses fantasmes. Ce «renversement dans le contraire», c'està-dire ce «passage d'une souffrance passive à une activité sadique » est évidemment ce qui explique la cruauté du futur révolutionnaire. Robespierre vit donc dans un état de fantasmatisation permanente. L'essentiel de ses fantasmes sont masochistes, mais aussi mégalomaniaques, tentant de compenser la terrible blessure narcissique infligée par son père. C'est pourquoi il écrit le mémoire sur les bâtards destiné à l'académie de Metz, car Maximilien se voulait le fruit d'une conception asexuée. Pour « survivre », l'enfant se construit des pères de substitution comme Rousseau. Le deuxième prénom de Robespierre est «d'ailleurs » [sic] Marie, vierge et mère...

Pour Robespierre, « la représentation de l'acte sexuel » est « insupportable ». Pour éviter la castration, il se réfugie dans l'indifférenciation des sexes. Il se représente lui-même " comme un être asexué et unique ». Artarit n'hésite pas à affirmer sans nuances : « Maximilien de Robespierre, malgré les madrigaux et les compliments compassés adressés aux femmes de son entourage, malgré le culte que lui vouèrent ses dévotes, n'eut à l'évidence [sic] aucune liaison, ni aucun commerce sexuel $»^{21}$. La problématique sexuelle de Robespierre comporte trois aspects : « l'impuissance psychique, la misogynie, et la défense contre l'homosexualité latente ». 
Or « la reconnaissance de ces dispositions affective de base est indispensable pour la compréhension de l'activité politique de Robespierre $»^{22}$. Arrêtons-nous là...

Critiquant pourtant explicitement la «méthode » psychanalytique d'Artarit en la qualifiant de «fatras théorique » et d'exemple «à ne pas suivre $»^{23}$, Laurent Dingli ne manque pas d'utiliser abondamment le ressort psychopathologique. Il note lui aussi que les « défaillances » de François Robespierre « déjà antérieures à son départ effectif », ont favorisé «chez son fils aîné la mise en place d'un idéal rigide, seule protection que le jeune Maximilien était en mesure d'opposer au vide affectif ».

La fameuse lettre à Buissart du 12 juin 1783 dans laquelle Robespierre raconte de manière ironique un de ses voyages à Carvin est l'occasion pour Dingli de se surpasser dans l'analyse de la psychologie de Robespierre. Cette lettre - que tous les spécialistes s'accordent à considérer comme une pochade dans laquelle Robespierre se moque des récits de voyage de son temps - est pour Dingli un document psychologique capital qu'il s'agit de prendre au premier degré. En effet, Robespierre étant "complètement hermétique à toute forme d'autodérision $»^{24}$, il n'est pas possible qu'il s'agisse d'une plaisanterie. CQFD. C'est pourquoi : " à travers sa prose indigente, il se montre tel qu'en lui-même, blessé, orgueilleux, toujours en quête d'une reconnaissance impossible. Il avoue ses obsessions, relate ses craintes et ses ambitions ». À propos du passage où Robespierre parle de manière (faussement admirative) de la statue du mayeur de Lens, justicier et médecin, Dingli écrit que

« si Robespierre manifeste une telle vénération pour l'ancien mayeur de Lens, c'est que ce dernier cumulait deux fonctions particulièrement importantes aux yeux du jeune avocat: la faculté de juger et celle de guérir. [...] Combattre le crime, c'est maintenir le corps sain de la nation et inversement. [...] Le moindre manquement à ces principes, la plus petite défaillance à ce dogme de pureté originelle seront alors considérés comme des tumeurs cancéreuses que le bistouri national se chargera de couper ».

On remarquera le brusque passage au temps futur, caractéristique de la « méthode » téléologique... Texte « d'une prétention stupéfiante », selon Dingli, la lettre à Buissart est « une véritable divagation » mégalomaniaque.

(22) Ibid., p. 68.

(23) Laurent DiNGLI, op. cit., p. 601.

(24) Ibid, p. 28. 
Robespierre raconte son arrivée triomphale à Carvin «pour compenser la blessure intolérable causée par les commis d'Arras » qui ne l'ont pas reconnu à la barrière de la ville ! Dans un des derniers paragraphes où Robespierre brode sur le plaisir de manger de la tarte arrageoise en bonne compagnie, Dingli voit l'ambition de Robespierre et le plaisir intense que lui procure la compagnie des petits notables locaux ${ }^{25}$. Une belle leçon de méthode de lecture des sources...

Dingli reprend également le thème du Robespierre asexué. C'est un ascète qui considère "l'expression des sens comme une véritable monstruosité, une souillure, une corruption ». Les femmes sont avant tout un moyen de parvenir et le support d'une reconnaissance toujours inassouvie. C'est pourquoi « Robespierre n'a, semble-t-il [sic], jamais eu de relation physique au cours de sa courte existence » et qu'il n'a jamais montré qu'un « désintérêt total pour cet acte vicieux et corrupteur » qu'est la relation sexuelle.

Robespierre est bien évidemment un paranoïaque manichéen qui voit le monde séparé entre les bons et les méchants, ce qui lui tient lieu de pensée politique ${ }^{26}$. Les méchants «n'apparaissent pas comme par magie, ils sont présents depuis l'enfance ». La condamnation de l'aristocratie et du clergé en 1789 relève déjà chez Robespierre d'une mentalité paranoïaque pathologique et d'essence totalitaire :

« Tout en se gardant des anachronismes [sic], on ne peut s'empêcher de penser aux métaphores antisémites employées en France et en Allemagne à la fin du XIX ${ }^{\mathrm{e}}$ siècle et dans la première moitié du $\mathrm{XX}^{\mathrm{e}}$ siècle. Le noble pour Sieyès ou Robespierre, le Juif pour les nazis, le patron ou le koulak pour les Soviétiques, l'intellectuel pour Pol Pot et Mao, tous les grands idéalistes ont eu leur repoussoir collectif, les éléments impurs à éliminer... »

Dans sa conclusion, Dingli insiste encore sur la dimension pathologique de la paranoïa chez Robespierre ${ }^{27}$. Le discours robespierriste

« appartenait à une personnalité de type paranoïde dont le discours était d'autant plus redoutable qu'il se fondait dans le réel. Une comparaison avec la personnalité d'Adolf Hitler ne semble pas incongrue sur ce point [sic], même si les conséquences ou les modalités du pouvoir détenu par ces deux hommes sont sans commune mesure. Toutefois, Hitler dont 
l'organisation mentale évoque étrangement et dans bien des domaines celle de l'Incorruptible, a su lui aussi adapter son discours à la réalité de l'Allemagne de $1933 »$.

Le thème pathologique « anti-totalitaire » se retrouve également chez Jean-François Fayard et ses 100 jours de Robespierre. Selon Fayard, la « réduction binaire du genre humain » est un « dispositif mental» particulier qui se trouve tout entier chez Robespierre dès avant 1789. La paranoïa s'accentue après la fuite du roi pour atteindre à un « dogmatisme schizophrène du totalitarisme d'État ». En l'an II, les mesures sociales sont le fruit d'une « volonté délirante du contrôle intégral de l'économie ». La Convention fait preuve d'un « acharnement pathologique » pour maîtriser les consciences. Dans ce cadre, Robespierre est le modèle de l'individu « psychorigide » doté d'une structure mentale utilisant la « dialectique » du complot « pour crédibiliser un mode de fonctionnement paranoïaque, une personnalité narcissique sachant habilement draper d'un discours tout en «prêt à penser » un délire du culte de la personnalité ${ }^{28}$. Même l'incorruptibilité de Robespierre n'est due qu'à une « rigidité pathologique l'abstrayant de toute réalité ${ }^{29} \gg$. (Et également au fait qu'il était aux frais de la riche famille Duplay ${ }^{30}$ !)

Enfin, dernier en date, Joël Schmidt (un écrivain ayant surtout produit des biographies de personnages du monde antique) s'illustre, lui aussi, dans le discours psychopathologique et « anti-totalitaire ». Certes, la logique de Maximilien n'est pas «démente » mais « chimérique », ce n'est pas par goût du sang que Robespierre agit mais par « la simple vénération de la vertu républicaine à l'ancienne ». Cette nouvelle version du conflit entre liberté des Anciens et des Modernes prend un tour franchement caricatural chez Schmidt, persuadé que Robespierre se prend vraiment pour Caton et Brutus réunis... Ainsi, en mai 1789, Robespierre « hanté par la gloire antique se voit davantage comme un sénateur romain habillé de sa toge et membre d'une assemblée qui ressemble au Sénat que comme un député ». Bien entendu, Schmidt ne doute pas que la sexualité de Robespierre n'ait été nulle, voire déviante. Pourtant, « on prétend » que Maximilien a vécu maritalement avec Éléonore Duplay, mais c'est impossible, car il n'a pas été « un homme couvert de femmes, ce qui aurait pu fort bien se produire, étant donné son extrême popularité ». Il est sûr qu'il a dû « recevoir des

(28) Jean-François FAYARD, op. cit., p. 43.

(29) Ibid, p. 114-115.

(30) Ibid., p. 160. 
propositions amoureuses et qu'il les a toujours repoussées ». «On » lui a attribué aussi des maîtresses mais « cet homme exècre le sexe, cela se sent [sic], cela se voit [re-sic], il l'a même certainement en horreur ${ }^{31} »$. C'est pourquoi l'accuser d'avoir eu des relations homosexuelles avec Saint-Just est absurde... La morale hétérosexuelle est sauve...

L'utilisation du thème anti-totalitaire devient franchement étrange sous la plume de Schmidt. À propos des hommages rendus à Robespierre et à Pétion par les Parisiens à la fin de la Constituante, il a ce passage étonnant :

«Les enfants qu'on présente à Robespierre, c'est un geste courant dans les régimes dictatoriaux, il n'est que de voir Hitler et Staline accomplissant les mêmes gestes en caressant les visages des enfants que les foules fanatiques leur tendent. Sans vouloir comparer les deux dictateurs à Robespierre [sic], notamment à cette date de sa carrière [re-sic] on éprouve tout de même un malaise devant tant de popularitée $e^{32} »$.

C'est bien pire après la mort de Louis XVI qui inaugure la Terreur [sic]. Robespierre perd alors la tête, il donne l'impression de ne plus «être maître de ce qu'il dit », il est en pleine « aliénation au sens psychiatrique du terme ${ }^{33}$. Conséquence : "submergé par une cécité politique qui frise le pathétique ou la folie, il se refuse à comprendre qu'il vit dans une autre époque que celle de la République romaine ${ }^{34} »$. Robespierre est donc « autiste », il dérive lentement mais sûrement vers le «crime contre l'humanité » qu'il aurait sûrement commis si les moyens techniques du XVIII ${ }^{\mathrm{e}}$ siècle avaient permis les exterminations de masse ${ }^{35}$, pourtant, quelques pages plus tard, Schmidt admet qu'il n'est quand même pas paranoïaque, car le «mot serait trop fort »... Donc Robespierre pas complètement fou, mais quand même pas très loin...

En septembre 2011 le magazine Historia vulgarise cette vulgate en publiant un numéro spécial intitulé «Robespierre. Le psychopathe légaliste $»^{36}$. Sous la plume du directeur de la rédaction Pierre Baron, on y apprend que Robespierre est un forcené, « enfermé dans une profonde solitude psychologique ». On notera le spectaculaire décalage entre les articles des historiens sérieux comme Bernard Gainot et Olivier Coquard

(31) Joël ScHMIDT, op. cit., p. 304.

(32) Ibid., p. 89.

(33) Ibid., p. 165.

(34) Ibid., p. 233

(35) Ibid., p. 229-230.

(36) Historia, septembre 2011, n 777. 
avec le ton donné par les chapeaux de la rédaction, même Max Gallo (celui de 2011, pas celui de la Lettre aux nouveaux Muscadins), interrogé par le journal, refuse l'expression de «maniaque de la guillotine » utilisé par Pierre Baron.

Il est évidemment impossible d'analyser (c'est le cas de le dire) ici les rapports problématiques entre psychanalyse, psychiatrie et histoire. Cette question est en dehors de notre domaine d'expertise. Mais rappelons quelques évidences (puisque le terme plait aux psychopathologues) : on ne peut rien savoir des soubassements enfantins de la psyché de Robespierre en dehors des sources directes et indirectes et ses discours nous renseignent davantage sur la culture politique de son temps que sur sa psychologie. Or les sources directes (les Euvres) n'en parlent jamais. Robespierre n'a jamais fait mention de ses parents, de son enfance, il évoque très rapidement ses années de collège dans un de ces discours... Il est vrai qu'il n'y a là rien d'étonnant, puisque nous n'avons pas plus de renseignements personnels sur la quasi-totalité des acteurs révolutionnaires. Les sources indirectes sont évidemment délicates à utiliser, il faut les contextualiser finement, voir à quels objectifs politiques elles correspondent, faire la part entre les discours des contemporains, ceux des Thermidoriens et ceux qui forment la trame de la biographie mythique de Robespierre. Peut-on se contenter de distribuer bons et mauvais points à l'abbé Proyart, à Montjoie, à Courtois, à Charlotte Robespierre en piochant telle ou telle anecdote dans leurs textes sans se demander si l'ensemble de la légende noire thermidorienne et de la légende dorée des robespierristes romantiques n'est tout simplement pas inutilisable pour élaborer une biographie ou une psychobiographie de Robespierre?

Nous n'avons aucune compétence sur les concepts, les spéculations psychanalytiques de Jean Artarit ou d'autres, en revanche, la méthode et le contenu historique des psychopathologues de Robespierre sont confondants de médiocrité. Toutes ces constructions ne reposent en dernière analyse que sur les préjugés et les impressions de leurs auteurs, et sur absolument rien d'autre.

Le but n'est pas de progresser dans la connaissance de Robespierre. Comme depuis deux siècles, il s'agit donc avant tout d'agiter le spectre Robespierre, de promener sa figure sur la scène, de la faire se mouvoir dans un décor de carton-pâte pour développer un discours de haine de la Révolution française. Le cas Robespierre est évidemment un observatoire particulièrement intéressant : Robespierre est un psychopathe, comme tous les révolutionnaires, ceux d'hier comme ceux d'aujourd'hui... Le discours pathologique n'est finalement qu'une manière à la mode de 
recycler le discours thermidorien. On ne dit plus que Robespierre inventait des complots imaginaires pour s'emparer du pouvoir suprême et devenir roi comme en l'an III, mais qu'il était paranoïaque, on ne dit plus que c'était un « monstre » mais qu'il était un « infirme affectif », etc. Sous la différence dans la rhétorique, c'est le même sempiternel discours réactionnaire qui vise à pathologiser les idées et les pratiques révolutionnaires...

\section{Biographies et travaux universitaires}

Dans la période considérée, seules trois biographies de Robespierre ont été publiées par des universitaires, historiens (Peter McPhee, Cécile Obligi) ou spécialiste des idées politiques (Ruth Scurr). Les objectifs des trois auteurs sont fort différents.

La récente synthèse grand public due à Cécile Obligi se situe clairement dans le camp de ceux qui entendent réhabiliter Robespierre et remettre en cause sa légende noire. L'auteur, conservatrice à la BNF, et qui a travaillé sur Bailly, se livre notamment à une critique serrée et salutaire des topoi négatifs sur le jeune Robespierre en relativisant les traits biographiques censés expliquer le parcours du révolutionnaire. Elle rappelle par exemple avec bon sens que la perte des parents est loin d'être un événement extraordinaire à la fin du $\mathrm{XVIII}^{\mathrm{e}}$ siècle, que la carrière arrageoise de Robespierre n'en fait pas un raté de l'Ancien Régime, que la prétendue paranoïa de Robespierre tient compte de l'existence de complots bien réels de la Contre-Révolution, etc. Par son format, (150 pages) ce petit livre se situe à la frontière de l'ouvrage très grand public et de l'ouvrage scientifique. Bien entendu, ce « portrait» ne saurait constituer une biographie érudite et complète de Robespierre, mais le parti pris de l'auteur qui laisse souvent la parole à Robespierre lui-même en fait une bonne introduction destinée au plus grand nombre.

Le propos de Ruth Scurr, enseignante en histoire des idées politiques à Cambridge, est plus ambitieux. Dans son Fatal Purity de 2006, elle a tenté de reprendre le chantier de la biographie de Robespierre en adoptant une approche «empathique » à l'égard de son sujet d'étude, mais malgré cette démarche a priori de bon aloi, l'auteur part du postulat que l'essentiel de la vie et de l'action de Robespierre est grosso modo connu, et surtout, elle reste dépendante des sources thermidoriennes et postérieures qu'elle ne livre pas à une critique contextualisée, mais à un tri en fonction de critères de vraisemblance qui mériteraient d'être explicités. Ruth Scurr reste, selon nous, d'une certaine manière prisonnière du piège biographique qui implique de combler les vides. Les citations apocryphes (Le « il ira 
loin, il croit tout ce qu'il dit » de Mirabeau), les témoignages thermidoriens (le portrait physique de Robespierre par Merlin de Thionville par exemple) sont utilisés avec la même valeur que les témoignages et les sources contemporaines pré-thermidoriennes.

Par ailleurs, le propos central de l'auteur est en phase avec le récit standard de la place de Robespierre dans la Révolution : Ruth Scurr cherche à situer le moment à partir duquel Robespierre a commencé à croire à l'image que renvoyait de lui la Révolution, à s'identifier à elle jusqu'à faire corps avec le cours des événements et finir par les incarner aux yeux de ses admirateurs et de ses contempteurs. Certes... mais les idées de «l'incarnation » de la Révolution - centrale chez Furet entre autres et le fait que Robespierre se serait «identifié » à la Révolution, sont en elles-mêmes fort discutables et nécessiteraient des développements. En partant d'un tel présupposé, Ruth Scurr s'aventure parfois sur des terres interprétatives bien connues : Robespierre «incarnant» la Révolution devient le « grand-prêtre » du culte de l'Être Suprême, il est à l'origine de la « loi infâme du 22 prairial », à la suite de laquelle Robespierre et la République s'identifièrent dans la «même tyrannie ». Robespierre croyait, « jusqu'à la folie », qu'il était « l'instrument de la providence », qu'il ne se trompait jamais, que tous ceux qui étaient en désaccord avec lui étaient des traîtres, etc. La pureté robespierriste, la vertu sont donc bien « fatales $»^{37} \ldots$

L'intérêt du livre de Ruth Scurr ne se limite pas à ces quelques remarques, mais malgré la tonalité empathique du récit, on est loin de la nouveauté dans l'approche de la figure de Robespierre...

L'ouvrage de l'historien australien Peter McPhee est d'une autre facture. Il s'agit cette fois du travail d'un spécialiste reconnu de l'histoire révolutionnaire. Deux éléments caractérisent cette nouvelle biographie : d'une part, l'auteur y fait la part belle aux années de formation ${ }^{38}$. D'autre part, il refuse la tyrannie du sens commun et s'attelle à déconstruire les mythes montés de toutes pièces par les adversaires de Robespierre dans la Révolution et au cours des périodes ultérieures.

La méthode choisie est celle de l'historien. Aux travaux psychologisants évoqués plus haut, McPhee oppose la rigueur historienne, la mesure et la circonspection face aux sources dont un grand nombre sont sujettes à caution. À ceux qui prétendent expliquer la trajectoire de Robespierre dans la Révolution par les effets traumatiques d'une enfance misérable

(37) Ruth SCURR, op. cit., p. 7, 10.

(38) Elles occupent 61 pages sur un texte total de 234 pages, soit un quart de l'ouvrage. 
et malheureuse, il répond simplement qu' « il n'existe pas de preuve pour corroborer de telles conclusions $»^{39}$. À ceux qui voient dans le leader jacobin un malade mental atteint de paranoïa narcissique obnubilé par l'existence de complots, l'historien répond que toutes les parties prenantes de la Révolution n'ont cessé de crier au complot à tour de rôle et démontre, preuves à l'appui, que les craintes de ce dernier n'étaient pas infondées ${ }^{40}$.

Maîtrisant mieux que d'autres auteurs la chronologie de la Révolution française, McPhee montre combien Robespierre, dans sa vie de révolutionnaire, est confronté à des problèmes de tous ordres qui surviennent sans discontinuer, qui redistribuent chaque jour les cartes du jeu politique et auxquels il faut apporter des réponses et s'adapter. Plus important encore, Mc Phee restitue à Robespierre l'humanité à laquelle il a droit, tout acteur historique incontournable soit-il. On comprendra mieux Robespierre, souligne-t-il dans sa conclusion, en le regardant d'abord comme un enfant et un jeune homme façonné autant par sa vie familiale que par le contexte social de l'Artois puis de Paris, et comme un jeune révolutionnaire embarqué, avec d'autres, par gros temps, dans la transformation radicale du monde ${ }^{41}$.

Si l'on excepte les trois ouvrages que nous venons d'évoquer, les travaux universitaires consacrés à Robespierre ces douze dernières années sont remarquablement peu nombreux : trois articles dans les $A H R F$ (Piquet, Rosso, Jacouty), trois dans d'autres revues (Guilhaumou, Seppinwall, Andress). Il faut y ajouter un chapitre du livre d'Antoine de Baecque. Les approches et les objets de ces contributions sont fort différents, mais la plupart (à l'exception de Jacques Guilhaumou) ont en commun de s'intéresser à Robespierre par le biais de thématiques plus générales que l'étude de son parcours politique proprement dit. Celle de JeanDaniel Piquet s'insère dans le débat initié par Yves Benot au moment du Bicentenaire et auquel ont participé notamment Florence Gauthier, Marcel Dorigny et Bernard Gainot sur les positions de Robespierre dans la question coloniale, l'article d'Alyssa Sepinwall se penche sur la nature $\mathrm{du}$ « féminisme » à travers le cas de Maximilien, celui de David Andress s'intéresse à la question du rapport entre sensibilité et engagement politique. Enfin, l'article de Jean-François Jacouty et le chapitre du livre d'Antoine de Baecque relèvent de l'historiographie (Louis Blanc et la construction du $d u e l$ Danton-Robespierre aux $\mathrm{XIX}^{\mathrm{e}}$ et $\mathrm{XX}^{\mathrm{e}}$ siècles) et non de l'étude du

(39) Peter McPHEE, op. cit., p.5.

(40) Ibid., p.110.

(41) Ibid., p.231. 
Robespierre historique. Finalement, seuls les articles de Maxime Rosso et surtout celui de Jacques Guilhaumou se focalisent sur le discours politique de Robespierre.

Prenant appui sur la parution du tome XI des Euvres, l'article de Jacques Guilhaumou est consacré à la formation du discours robespierriste dans les années 1780. D'une certaine manière, il rejoint le souci de Peter McPhee de cerner la construction de la position de Robespierre comme commentateur et acteur politique, c'est-à-dire « sur la manière dont Robespierre se constitue lui-même comme sujet observant, participant et agissant dans les années $1780^{42}$ ». Jacques Guilhaumou apporte ainsi une contribution importante à la compréhension de la formation du révolutionnaire comme sujet politique et comme " politique philosophe », mais aussi comme sujet sensible dans le cadre du débat sur l'état social. C'est par ce travail que Robespierre élabore sa vision d'une société juste fondée sur la liberté et les droits naturels des hommes, mais aussi sa conception du « peuple », compris comme la « dernière classe de la société ». C'est par la demande de réforme des «mœurs » que le «politique philosophe » prend position et se constitue plus tard comme « porte-parole » et « représentant».

La production éditoriale sur Robespierre est également marquée par la publication continue d'anthologies et d'éditions de ses discours. L'édition critique de A la nation artésienne par Marie-Laure Legay est un travail important qui montre la manière dont Robespierre articule revendications locales et universelles. Nous sommes ici encore dans ce que Jacques Guilhaumou a appelé « l'ontologie du discours robespierriste ». La publication des Euvres s'est poursuivie en 2007 avec le tome XI présenté par Florence Gauthier, également éditrice d'une anthologie avec Yannick Bosc et Sophie Wahnich en 2000. Dans la continuité de leurs travaux antérieurs, les trois historiens - également animateurs du site Révolutionfrançaise.net qui accorde une large place à des études sur Robespierre insistent sur « l'actualité d'un homme politique irrécupérable » et sur la centralité du droit naturel dans les conceptions et l'action de Robespierre.

Ce très rapide tour d'horizon fait apparaître une autre caractéristique de la production éditoriale depuis le Bicentenaire: on peut presque parler « d'évitement » de la part des historiens de l'école dite jacobine (à l'exception de Claude Mazauric ${ }^{43}$, Florence Gauthier, Françoise Brunel ou

(42) Jacques Guilhaumou, op. cit., p. 2.

(43) Voir notamment son introduction à l'édition du Centenaire de la SER des CEuvres complètes de Robespierre, ainsi que sa participation au numéro de la revue Commune, numéro 45, avril 2007, qui contient également un article de Georges Labica. 
de Jacques Guilhaumou et de leurs élèves) qui ont délaissé le personnage de Robespierre pour se consacrer à d'autres champs de l'historiographie de la Révolution : histoire culturelle, des formes de participation politique, des institutions locales, de la Contre-Révolution, etc. Alors que la biographie a opéré un retour avec les travaux de Pierre Serna sur Antonelle, de Michel Biard sur Collot d'Herbois ou de Christine Le Bozec sur Boissy d'Anglas, la dernière biographie consacrée à Robespierre est pourtant due à un historien australien et pas à un historien « robespierriste » français...

Pourquoi cet «évitement» de la part d'historiens, pour la plupart membres de la Société des études robespierristes? La première raison est peut-être qu'Albert Mathiez n'a pas réellement eu d'héritier historiographique et que la tradition jauressienne d'histoire sociale, pourtant très présente jusqu'à aujourd'hui, a en partie tourné le dos à l'histoire mathiezienne des idées politiques. Une deuxième raison est peut-être qu'ils ont abandonné un terrain sur lequel il était difficile d'intervenir scientifiquement tant les ouvrages médiocres pullulent. Passer son temps à la réfutation des erreurs innombrables des pamphlets haineux sur Robespierre est une activité ingrate. Par ailleurs, dans la dynamique des commémorations suivant le Bicentenaire, de nombreux travaux et colloques ont été consacrés à la période autrefois délaissée du Directoire et le nombre de travaux sur la période 1789-1794 a décru. Mais le contexte semble avoir changé tout récemment. L'affaire des manuscrits et la publication d'un ouvrage collectif grand public, intitulé Robespierre, portraits croisés regroupant une série de synthèses thématiques ${ }^{44}$ en est peut-être l'indice, et l'on annonce une nouvelle biographie de Robespierre chez Fayard confiée à Hervé Leuwers.

\section{Conclusion}

En 1965, Jacques Godechot estimait qu'une forme de consensus s'était réalisée sur Robespierre dans l'historiographie républicaine et que les discours haineux de la Contre-Révolution n'étaient plus qu'un épiphénomène ${ }^{45}$. Excellent historien, Godechot s'est, hélas, montré très mauvais prophète! Dans les années 1970, le livre d'Alice Gérard sur l'historiographie de la Révolution montrait pourtant encore que la figure de Robespierre faisait toujours clivage et qu'elle restait un enjeu fondamental

(44) Michel BIARD, Philippe BoURdin (dir.), Robespierre, portraits croisés, Paris, Armand Colin, 2012.

(45) Actes du Colloque Robespierre, Paris, Société des études robespierristes, 1967. 
de l'historiographie de la Révolution ${ }^{46}$. L'actualité récente de Robespierre prouve - parfois en creux - que la connaissance de l'action et de la pensée de l'Incorruptible est loin d'avoir épuisé son potentiel. Les travaux universitaires de la dernière décennie ont insisté sur la formation de Robespierre, mais il reste encore beaucoup à faire : le républicanisme de Robespierre, sa conception de l'état social, sa position de législateurphilosophe, sa place dans toute une série de débats relativement peu étudiés ces derniers temps (par exemple ses projets de réforme de la justice), autant de thèmes et bien d'autres encore pourraient faire l'objet d'études nouvelles.

Marc BELISSA

Université Paris Ouest Nanterre - CHISCO

UFR SSA, Département d'histoire 200 Avenue de la République 92000 Nanterre mbelissa@club-internet.fr

Julien LOUVRIER University of Helsinki Department of Philosophy, History, Culture and Art Studies julien.louvrier@gmail.com 